\title{
A NEW QUANTITATIVE TWO WEIGHT THEOREM FOR THE HARDY-LITTLEWOOD MAXIMAL OPERATOR
}

\author{
CARLOS PÉREZ AND EZEQUIEL RELA
}

(Communicated by Alexander Iosevich)

\begin{abstract}
A quantitative two weight theorem for the Hardy-Littlewood maximal operator is proved, improving the known ones. As a consequence, a new proof of the main results in papers by Hytönen and the first author and Hytönen, the first author and Rela is obtained which avoids the use of the sharp quantitative reverse Holder inequality for $A_{\infty}$ proved in those papers. Our results are valid within the context of spaces of homogeneous type without imposing the non-empty annuli condition.
\end{abstract}

\section{INTRODUCTION AND MAIN RESULTS}

1.1. Introduction. The purpose of this note is to present a quantitative two weight theorem for the Hardy-Littlewood maximal operator when the underlying space is a space of homogeneous type $\mathcal{S}$ (SHT in the sequel), endowed with a quasimetric $\rho$ and a doubling measure $\mu$ (see Section 2.1 for the precise definitions). We briefly recall some background on this problem in the euclidean or classical setting, when we are working in $\mathbb{R}^{n}$ and we consider Lebesgue measure and euclidean metric. We also assume that in this classical setting all the maximal operators involved and $A_{p}$ classes of weights are defined over cubes. Let $M$ stand for the usual uncentered Hardy-Littlewood maximal operator:

$$
M f(x)=\sup _{Q \ni x} \frac{1}{|Q|} \int_{Q}|f| d x .
$$

The problem of characterizing the pair of weights for which the maximal operator is bounded between weighted Lebesgue spaces was solved by Sawyer Saw82]: To be more precise, if $1<p<\infty$ we define for any pair of weights $w, \sigma$, the (two weight) norm,

$$
\|M(\cdot \sigma)\|_{L^{p}(w)}:=\sup _{f \in L^{p}(\sigma)} \frac{\|M(f \sigma)\|_{L^{p}(w)}}{\|f\|_{L^{p}(\sigma)}},
$$

then Sawyer showed that $\|M(\cdot \sigma)\|_{L^{p}(w)}$ is finite if and only if

$$
\sup _{Q} \frac{\int_{Q}\left(M\left(\chi_{Q} \sigma\right)^{p} w d x\right.}{\sigma(Q)}<\infty,
$$

Received by the editors April 26, 2013.

2010 Mathematics Subject Classification. Primary 42B25; Secondary 43A85.

Key words and phrases. Two weight theorem, space of homogeneous type, Muckenhoupt weights, Calderón-Zygmund, maximal functions.

Both authors were supported by the Spanish Ministry of Science and Innovation grant MTM2012-30748 and by the Junta de Andalucía, grant FQM-4745. 
where the supremum is taken over all the cubes in $\mathbb{R}^{n}$. A quantitative precise version of this result is the following: if we define

$$
[w, \sigma]_{S_{p}}:=\left(\frac{1}{\sigma(Q)} \int_{Q} M\left(\sigma \chi_{Q}\right)^{p} w d x\right)^{1 / p},
$$

then

$$
\|M(\cdot \sigma)\|_{L^{p}(w)} \sim p^{\prime}[w, \sigma]_{S_{p}},
$$

where $\frac{1}{p}+\frac{1}{p^{\prime}}=1$. This result is due to K. Moen and can be found in [Moe09.

However, it is still an open problem to find a characterization more closely related to the $A_{p}$ condition of Muckenhoupt which is easier to use in applications. Indeed, recall that the two weight $A_{p}$ condition:

$$
\sup _{Q}\left(f_{Q} w d x\right)\left(f_{Q} v^{-\frac{1}{p-1}} d x\right)^{p-1}<\infty
$$

is necessary for the boundedness of $M$ from $L^{p}(v)$ into $L^{p}(w)$ (which is clearly equivalent, setting $\sigma=v^{1-p^{\prime}}$, to the two weight problem), but it is not sufficient. Therefore, the general idea is to strengthen the $A_{p}$ condition to make it sufficient. The first result in this direction is due to Neugebauer [Neu83, proving that, for any $r>1$, it is sufficient to consider the following "power bump" for the $A_{p}$ condition:

$$
\sup _{Q}\left(f_{Q} w^{r} d x\right)^{\frac{1}{r}}\left(f_{Q} v^{-\frac{r}{p-1}} d x\right)^{(p-1) r}<\infty .
$$

Later, the first author improved this result in Pér95 by considering a different approach which allowed consideration of much larger classes weights. The new idea is to replace only the average norm associated to the weight $v^{-\frac{1}{p-1}}$ in (1.3) by a "stronger" norm which is often called a "bump". This norm is defined in terms of an appropriate Banach function $X$ space satisfying certain special property. This property is related to the $L^{p}$ boundedness of a natural maximal function related to the space. More precisely, for a given Banach function space $X$, the local $X$-average of a measurable function $f$ associated to the cube $Q$ is defined as

$$
\|f\|_{X, Q}=\left\|\tau_{\ell(Q)}\left(f \chi_{Q}\right)\right\|_{X},
$$

where $\tau_{\delta}$ is the dilation operator $\tau_{\delta} f(x)=f(\delta x), \delta>0$ and $\ell(Q)$ stands for the sidelength of the cube $Q$. The natural maximal operator associated to the space $X$ is defined as

$$
M_{X} f(x)=\sup _{Q: x \in Q}\|f\|_{X, Q}
$$

and the key property is that the maximal operator $M_{X^{\prime}}$ is bounded on $L^{p}\left(\mathbb{R}^{n}\right)$ where $X^{\prime}$ is the associate space to $X$ (see (1.5) below).

As a corollary of our main result, Theorem 1.3 we will give a quantitative version of the main result from [Pér95] regarding sufficient conditions for the two weight inequality to hold:

Theorem 1.1. Let $w$ and $\sigma$ be a pair of weights that satisfies the condition

$$
\sup \left(f_{Q} w d x\right)\left\|\sigma^{1 / p^{\prime}}\right\|_{X, Q}^{p}<\infty .
$$


Suppose, in addition, that the maximal operator associated to the associate space is bounded on $L^{p}\left(\mathbb{R}^{n}\right)$ :

$$
M_{X^{\prime}}: L^{p}\left(\mathbb{R}^{n}\right) \rightarrow L^{p}\left(\mathbb{R}^{n}\right) .
$$

Then there is a finite positive constant $C$ such that:

$$
\|M(\cdot \sigma)\|_{L^{p}(w)} \leq C .
$$

In this note we give a different result of this type with the hope that it may lead to different, possibly better, conditions for the two weight problem for Singular Integral Operators.

Most of the interesting examples are obtained when $X$ is an Orlicz space $L_{\Phi}$ defined in terms of the Young function $\Phi$ (see Section 2.1 for the precise definitions). In this case, the local average with respect to $\Phi$ over a cube $Q$ is

$$
\|f\|_{\Phi, Q}=\|f\|_{\Phi, Q, \mu}=\inf \left\{\lambda>0: \frac{1}{\mu(Q)} \int_{Q} \Phi\left(\frac{|f|}{\lambda}\right) d x \leq 1\right\}
$$

where here $\mu$ is the Lebesgue measure. The corresponding maximal function is

$$
M_{\Phi} f(x)=\sup _{Q: x \in Q}\|f\|_{\Phi, Q} .
$$

Related to condition (1.4) here we introduce the following quantities.

Definition 1.2. Let $(\mathcal{S}, d \mu)$ be an SHT. Given a ball $B \subset \mathcal{S}$, a Young function $\Phi$ and two weights $w$ and $\sigma$, we define the quantity

$$
A_{p}(w, \sigma, B, \Phi):=\left(f_{B} w d \mu\right)\left\|\sigma^{1 / p^{\prime}}\right\|_{\Phi, B}^{p}
$$

and we say that a pair of weights belongs to the $A_{p, \Phi}$ class if

$$
[w, \sigma, \Phi]_{A_{p}}:=\sup _{B} A_{p}(w, \sigma, B, \Phi)<\infty
$$

where the sup is taken over all balls in the space. In the particular case of $\Phi(t)=t^{p^{\prime}}$, this condition corresponds to the classical $A_{p}$ condition and we use the notation

$$
[w, \sigma]_{A_{p}}:=\sup _{B}\left(f_{B} w d \mu\right)\left(f_{B} \sigma d \mu\right)^{p-1} .
$$

Now we define a generalization of the Fuji-Wilson constant of an $A_{\infty}$ weight $\sigma$ as introduced in $\mathrm{HP}$ by means of a Young function $\Phi$ :

$$
[\sigma, \Phi]_{W_{p}}:=\sup _{B} \frac{1}{\sigma(B)} \int_{B} M_{\Phi}\left(\sigma^{1 / p} \chi_{B}\right)^{p} d \mu .
$$

Note that the particular choice of $\Phi_{p}(t):=t^{p}$ reduces to the $A_{\infty}$ constant (see (2.5) from Section 2.1):

$$
\left[\sigma, \Phi_{p}\right]_{W_{p}}=\sup _{B} \frac{1}{\sigma(B)} \int_{B} M\left(\sigma \chi_{B}\right) d \mu=[\sigma]_{A_{\infty}} .
$$


1.2. Main results. Our main purpose in the present note is to address the problem mentioned above within the context of spaces of homogeneous type. In this context, the Hardy-Littlewood maximal operator $M$ is defined over balls:

$$
M f(x)=\sup _{B \ni x} \frac{1}{\mu(B)} \int_{B}|f| d \mu .
$$

The Orlicz type maximal operators are defined also with balls and with respect to the measure $\mu$ in the natural way.

Our main result is the following theorem.

Theorem 1.3. Let $1<p<\infty$ and let $\Phi$ be any Young function with conjugate function $\bar{\Phi}$. Then, for any pair of weights $w, \sigma$, there exists a structural constant $C>0$ such that the (two weight) norm defined in (1.1) satisfies

$$
\|M(\cdot \sigma)\|_{L^{p}(w)} \leq C p^{\prime}\left([w, \sigma, \Phi]_{A_{p}}[\sigma, \bar{\Phi}]_{W_{p}}\right)^{1 / p} .
$$

We emphasize that (1.10), which is even new in the usual context of Euclidean Spaces, fits into the spirit of the $A_{p}-A_{\infty}$ theorem derived in [HP] and [HPR12]. The main point here is that we have a two weight result with a better condition and with a proof that avoids completely the use of the sharp quantitative reverse Hölder inequality for $A_{\infty}$ weights proved in these papers. This property is, of course, of independent interest but it is not used in our results.

From this theorem, we derive several corollaries. First, we have a direct proof of the two weight result derived in $\left[\mathrm{HP}\right.$ using the $[w]_{A_{\infty}}$ constant of Fujii-Wilson (2.5).

Corollary 1.4. Under the same hypothesis of Theorem 1.3, we have that there exists a structural constant $C>0$ such that

$$
\|M(\cdot \sigma)\|_{L^{p}(w)} \leq C p^{\prime}\left([w, \sigma]_{A_{p}}[\sigma]_{A_{\infty}}\right)^{1 / p} .
$$

Note that the result in Theorem 1.3 involves two suprema as in Corollary 1.4. It would be interesting to find out if there is a version of this result involving only one supremum. There is some evidence that it could be the case; see for example $[\mathrm{HP}$, Theorem 4.3. See also the recent work [LM].

As a second consequence of Theorem 1.3, we have the announced quantitative version of Theorem 1.1;

Corollary 1.5. Under the same hypothesis of Theorem 1.3 , we have that there exists a structural constant $C>0$ such that

$$
\|M(\cdot \sigma)\|_{L^{p}(w)} \leq C p^{\prime}[w, \sigma, \Phi]_{A_{p}}^{1 / p}\left\|M_{\bar{\Phi}}\right\|_{L^{p}\left(\mathbb{R}^{n}\right)} .
$$

We remark that this approach produces a non-optimal dependence on $p$, since we have to pay with one $p^{\prime}$ for using Sawyer's theorem. However, the ideas from the proof of Theorem 1.3 can be used to derive a direct proof of Corollary 1.5 without the $p^{\prime}$ factor. We include the proof in the appendix.

Finally, for the one weight problem, we recover the known mixed bound.

Corollary 1.6. For any $A_{p}$ weight $w$ the following mixed bound holds:

$$
\|M\|_{L^{p}(w)} \leq C p^{\prime}\left([w]_{A_{p}}[\sigma]_{A_{\infty}}\right)^{1 / p}
$$

where $C$ is a structural constant and as usual $\sigma=w^{1-p^{\prime}}$ is the dual weight. 
Remark 1.7. To be able to extend the proofs to this general scenario, we need to use (and prove) suitable versions of classical tools on this subject, such as CalderónZygmund decompositions. We remark that in previous works ([PW01, SW92]) most of the results are proved under the assumption that the space has non-empty annuli. The main consequence of this property is that in that case the measure $\mu$ enjoys a reverse doubling property, which is crucial in the proof of CalderónZygmund type lemmas. However, this assumption implies, for instance, that the space has infinite measure and no atoms (i.e. points with positive measure) and therefore constraints the family of spaces under study. Recently, some of those results were proven without this hypothesis; see for example PS04]. Here we choose to work without the annuli property and therefore we need to adapt the proofs from PW01. Hence, we will need to consider separately the cases when the space has finite or infinite measure. An important and useful result on this matter is the following:

Lemma 1.8 (GGKK98]). Let $(\mathcal{S}, \rho, \mu)$ be a space of homogeneous type. Then $\mathcal{S}$ is bounded if and only if $\mu(\mathcal{S})<\infty$.

1.3. Outline. The article is organized as follows. In Section 2 we summarize some basic needed results on spaces of homogeneous type and Orlicz spaces. We also include a Calderón-Zygmund type decomposition lemma. In Section 3 we present the proofs of our results. Finally, we include in Section 4 an Appendix with a direct proof of a slightly better result than Corollary 1.4 .

\section{Preliminaries}

In this section we first summarize some basic aspects regarding spaces of homogeneous type and Orlicz spaces. Then, we include a Calderón-Zygmund (C-Z) decomposition lemma adapted to our purposes.

2.1. Spaces of homogeneous type. A quasimetric $d$ on a set $\mathcal{S}$ is a function $d: \mathcal{S} \times \mathcal{S} \rightarrow[0, \infty)$ which satisfies

(1) $d(x, y)=0$ if and only if $x=y$;

(2) $d(x, y)=d(y, x)$ for all $x, y$;

(3) there exists a finite constant $\kappa \geq 1$ such that, for all $x, y, z \in \mathcal{S}$,

$$
d(x, y) \leq \kappa(d(x, z)+d(z, y)) .
$$

Given $x \in \mathcal{S}$ and $r>0$, we define the ball with center $x$ and radius $r, B(x, r):=$ $\{y \in \mathcal{S}: d(x, y)<r\}$ and we denote its radius $r$ by $r(B)$ and its center $x$ by $x_{B}$. A space of homogeneous type $(\mathcal{S}, d, \mu)$ is a set $\mathcal{S}$ endowed with a quasimetric $d$ and a doubling non-negative Borel measure $\mu$ such that

$$
\mu(B(x, 2 r)) \leq C \mu(B(x, r)) .
$$

Let $C_{\mu}$ be the smallest constant satisfying (2.1). Then $D_{\mu}=\log _{2} C_{\mu}$ is called the doubling order of $\mu$. It follows that

$$
\frac{\mu(B)}{\mu(\tilde{B})} \leq C_{\mu}^{2+\log _{2} \kappa}\left(\frac{r(B)}{r(\tilde{B})}\right)^{D_{\mu}} \text { for all balls } \tilde{B} \subset B .
$$

In particular for $\lambda>1$ and $B$ a ball, we have that

$$
\mu(\lambda B) \leq(2 \lambda)^{D_{\mu}} \mu(B) .
$$


Here, as usual, $\lambda B$ stands for the dilation of a ball $B(x, \lambda r)$ with $\lambda>0$. Throughout this paper, we will say that a constant $c=c(\kappa, \mu)>0$ is a structural constant if it depends only on the quasimetric constant $\kappa$ and the doubling constant $C_{\mu}$.

An elementary but important property of the quasimetric is the following. Suppose that we have two balls $B_{1}=B\left(x_{1}, r_{1}\right)$ and $B_{2}=B\left(x_{2}, r_{2}\right)$ with non-empty intersection. Then,

$$
r_{1} \leq r_{2} \Longrightarrow B_{1} \subset \kappa(2 \kappa+1) B_{2} \text {. }
$$

This is usually known as the "engulfing" property and follows directly from the quasitriangular property of the quasimetric.

In a general space of homogeneous type, the balls $B(x, r)$ are not necessarily open, but by a theorem of Macias and Segovia MS79, there is a continuous quasimetric $d^{\prime}$ which is equivalent to $d$ (i.e., there are positive constants $c_{1}$ and $c_{2}$ such that $c_{1} d^{\prime}(x, y) \leq d(x, y) \leq c_{2} d^{\prime}(x, y)$ for all $\left.x, y \in \mathcal{S}\right)$ for which every ball is open. We always assume that the quasimetric $d$ is continuous and that balls are open.

We will adopt the usual notation: if $\nu$ is a measure and $E$ is a measurable set, $\nu(E)$ denotes the $\nu$-measure of $E$. Also, if $f$ is a measurable function on $(\mathcal{S}, d, \mu)$ and $E$ is a measurable set, we will use the notation $f(E):=\int_{E} f(x) d \mu$. We also will denote the $\mu$-average of $f$ over a ball $B$ as $f_{B}=f_{B} f d \mu$. We recall that a weight $w$ (any non-negative measurable function) satisfies the $A_{p}$ condition for $1<p<\infty$ if

$$
[w]_{A_{p}}:=\sup _{B}\left(f_{B} w d \mu\right)\left(f_{B} w^{-\frac{1}{p-1}} d \mu\right)^{p-1},
$$

where the supremum is taken over all the balls in $\mathcal{S}$. The $A_{\infty}$ class is defined in the natural way by $A_{\infty}:=\bigcup_{p>1} A_{p}$.

This class of weights can also be characterized by means of an appropriate constant. In fact, there are various different definitions of this constant, all of them equivalent in the sense that they define the same class of weights. Perhaps the more classical and known definition is the following due to Hruščev Hru84, (see also GCRdF85]):

$$
[w]_{A_{\infty}}^{\exp }:=\sup _{B}\left(f_{B} w d \mu\right) \exp \left(f_{B} \log w^{-1} d \mu\right) .
$$

However, in $\left[\mathrm{HP}\right.$ the authors use a "new" $A_{\infty}$ constant (which was originally introduced implicitly by Fujii in Fuj78 and later by Wilson in Wil87]), which seems to be better suited. For any $w \in A_{\infty}$, we define

$$
[w]_{A_{\infty}}:=[w]_{A_{\infty}}^{W}:=\sup _{B} \frac{1}{w(B)} \int_{B} M\left(w \chi_{B}\right) d \mu
$$

where $M$ is the usual Hardy-Littlewood maximal operator. When the underlying space is $\mathbb{R}^{d}$, it is easy to see that $[w]_{A_{\infty}} \leq c[w]_{A_{\infty}}^{\exp }$ for some structural $c>0$. In fact, it is shown in $\left[\mathrm{HP}\right.$ that there are examples showing that $[w]_{A_{\infty}}$ is much smaller than $[w]_{A_{\infty}}^{\exp }$. The same line of ideas yields the inequality in this wider scenario. See the recent work of Beznosova and Reznikov $[\mathrm{BR}]$ for a comprehensive and thorough study of these different $A_{\infty}$ constants. We also refer the reader to the forthcoming work of Duoandikoetxea, Martin-Reyes and Ombrosi [DMRO13] for a discussion regarding different definitions of $A_{\infty}$ classes. 
2.2. Orlicz spaces. Here we recall some basic definitions and facts about Orlicz spaces.

A function $\Phi:[0, \infty) \rightarrow[0, \infty)$ is called a Young function if it is continuous, convex, increasing and satisfies $\Phi(0)=0$ and $\Phi(t) \rightarrow \infty$ as $t \rightarrow \infty$. For Orlicz spaces, we are usually only concerned about the behavior of Young functions for $t$ large. The space $L_{\Phi}$ is a Banach function space with the Luxemburg norm

$$
\|f\|_{\Phi}=\|f\|_{\Phi, \mu}=\inf \left\{\lambda>0: \int_{\mathcal{S}} \Phi\left(\frac{|f|}{\lambda}\right) d \mu \leq 1\right\} .
$$

Each Young function $\Phi$ has an associated complementary Young function $\bar{\Phi}$ satisfying

$$
t \leq \Phi^{-1}(t) \bar{\Phi}^{-1}(t) \leq 2 t
$$

for all $t>0$. The function $\bar{\Phi}$ is called the conjugate of $\Phi$, and the space $L_{\bar{\Phi}}$ is called the conjugate space of $L_{\Phi}$. For example, if $\Phi(t)=t^{p}$ for $1<p<\infty$, then $\bar{\Phi}(t)=t^{p^{\prime}}, p^{\prime}=p /(p-1)$, and the conjugate space of $L^{p}(\mu)$ is $L^{p^{\prime}}(\mu)$.

A very important property of Orlicz spaces is the generalized Hölder inequality

$$
\int_{\mathcal{S}}|f g| d \mu \leq 2\|f\|_{\Phi}\|g\|_{\bar{\Phi}}
$$

Now we introduce local versions of Luxemburg norms. If $\Phi$ is a Young function, let

$$
\|f\|_{\Phi, B}=\|f\|_{\Phi, B, \mu}=\inf \left\{\lambda>0: \frac{1}{\mu(B)} \int_{B} \Phi\left(\frac{|f|}{\lambda}\right) d \mu \leq 1\right\} .
$$

Furthermore, the local version of the generalized Hölder inequality (2.6) is

$$
\frac{1}{\mu(B)} \int_{B} f g d \mu \leq 2\|f\|_{\Phi, B}\|g\|_{\bar{\Phi}, B} .
$$

Recall the definition of the maximal type operators $M_{\Phi}$ from (1.6):

$$
M_{\Phi} f(x)=\sup _{B: x \in B}\|f\|_{\Phi, B}
$$

An important fact related to this sort of operator is that its boundedness is related to the so-called $B_{p}$ condition. For any positive function $\Phi$ (not necessarily a Young function), we have that

$$
\left\|M_{\Phi}\right\|_{L^{p}(\mathcal{S})}^{p} \leq c_{\mu, \kappa} \alpha_{p}(\Phi)
$$

where $\alpha_{p}(\Phi)$ is the following tail condition:

$$
\alpha_{p}(\Phi)=\int_{1}^{\infty} \frac{\Phi(t)}{t^{p}} \frac{d t}{t}<\infty .
$$

It is worth noting that in the recent article [LL the authors define the appropriate analogue of the $B_{p}$ condition in order to characterize the boundedness of the strong Orlicz-type maximal function defined over rectangles both in the linear and multilinear cases. Recent developments and improvements can also be found in $\mathrm{MP}$, where the authors addressed the problem of studying the maximal operator between Banach function spaces. 
2.3. Calderón-Zygmund decomposition for spaces of homogeneous type. The following lemma is a classical result in the theory, regarding a decomposition of a generic level set of the Hardy-Littlewood maximal function $M$. Some variants can be found in AM84 for $M$ and in Aim85 for the centered maximal function $M^{c}$. In this latter case, the proof is straightforward. Here we include a detailed proof for the general case of $M$ where some extra subtleties are needed.

Lemma 2.1 (Calderón-Zygmund decomposition). Let $B$ be a fixed ball and let $f$ be a bounded non-negative measurable function. Let $M$ be the usual non-centered Hardy-Littlewood maximal function. Define the set $\Omega_{\lambda}$ as

$$
\Omega_{\lambda}=\{x \in B: M f(x)>\lambda\} .
$$

Let $\lambda>0$ be such that $\lambda \geq f_{B} f d \mu$. If $\Omega_{\lambda}$ is non-empty, then given $\eta>1$, there exists a countable family $\left\{B_{i}\right\}$ of pairwise disjoint balls such that, for $\theta=4 \kappa^{2}+\kappa$,

i) $\bigcup_{i} B_{i} \subset \Omega_{\lambda} \subset \bigcup_{i} \theta B_{i}$.

ii) For all $i$,

$$
\lambda<\frac{1}{\mu\left(B_{i}\right)} \int_{B_{i}} f d \mu .
$$

iii) If $B$ is any ball such that $B_{i} \subset B$ for some $i$ and $r(B) \geq \operatorname{\eta r}\left(B_{i}\right)$, we have that

$$
\frac{1}{\mu(B)} \int_{B} f d \mu \leq \lambda
$$

Proof. Define, for each $x \in \Omega_{\lambda}$, the following set:

$$
\mathcal{R}_{x}^{\lambda}=\left\{r>0: f_{B} f d \mu>\lambda, x \in B=B(y, r)\right\},
$$

which is clearly non-empty. The key here is to prove that $\mathcal{R}_{x}^{\lambda}$ is bounded. If the whole space is bounded, there is nothing to prove. In the case of unbounded spaces, we argue as follows. Since the space is of infinite measure (recall Lemma 1.8), and clearly $S=\bigcup_{r>0} B(x, r)$, we have that $\mu(B(x, r))$ goes to $+\infty$ when $r \rightarrow \infty$ for any $x \in \mathcal{S}$. Therefore, for $K=\kappa(2 \kappa+1)$, we can choose $r_{1}$ such that the ball $B_{1}=B\left(x, r_{1}\right)$ satisfies the inequality

$$
\mu\left(B_{1}\right) \geq \frac{2(2 K)^{D_{\mu}}\|f\|_{L^{1}}}{\lambda} .
$$

Suppose now that $\sup \mathcal{R}_{x}^{\lambda}=+\infty$. Then we can choose a ball $B_{2}=B\left(y, r_{2}\right)$ for some $y$ such that $x \in B_{2}, f_{B_{2}} f d \mu>\lambda$ and $r_{2}>r_{1}$. Now, by the engulfing property (2.4), we obtain that $B_{1} \subset K B_{2}$. The doubling condition (2.3) yields

$$
\mu\left(B_{1}\right) \leq \mu\left(K B_{2}\right) \leq(2 k)^{D_{\mu}} \mu\left(B_{2}\right) .
$$

Then we obtain that

$$
\frac{2\|f\|_{L^{1}}}{\lambda} \leq \mu\left(B_{2}\right)<\frac{\|f\|_{L^{1}}}{\lambda}
$$

which is a contradiction. We conclude that, in any case, for any $x \in \Omega_{\lambda}$, we have that $\sup \mathcal{R}_{x}^{\lambda}<\infty$.

Now fix $\eta>1$. If $x \in \Omega_{\lambda}$, there is a ball $B_{x}$ containing $x$, whose radius $r\left(B_{x}\right)$ satisfies $\frac{\sup \mathcal{R}_{x}^{\lambda}}{\eta}<r\left(B_{x}\right) \leq \sup \mathcal{R}_{x}^{\lambda}$, and for which $f_{B_{x}} f d \mu>\lambda$. Thus the ball $B_{x}$ satisfies ii) and iii). Also note that $\Omega_{\lambda}=\bigcup_{x \in \Omega_{\lambda}} B_{x}$. Picking a Vitali type subcover 
of $\left\{B_{x}\right\}_{x \in \Omega_{\lambda}}$ as in [SW92], Lemma 3.3, we obtain a family of pairwise disjoint balls $\left\{B_{i}\right\} \subset\left\{B_{x}\right\}_{x \in \Omega_{\lambda}}$ satisfying i). Therefore $\left\{B_{i}\right\}$ satisfies i), ii) and iii).

We will need another important lemma, in order to handle simultaneously decompositions of level sets at different scales.

Lemma 2.2. Let $B$ be a ball and let $f$ be a bounded non-negative measurable function. Let also $a \gg 1$ and, for each integer $k$ such that $a^{k}>f_{B} f d \mu$, we define $\Omega_{k}$ as

$$
\Omega_{k}=\left\{x \in B: M f(x)>a^{k}\right\} .
$$

Let $\left\{E_{i}^{k}\right\}_{i, k}$ be defined by $E_{i}^{k}=B_{i}^{k} \backslash \Omega_{k+1}$, where the family of balls $\left\{B_{i}^{k}\right\}_{i, k}$ is obtained by applying Lemma 2.1 to each $\Omega_{k}$. Then, for $\theta=4 \kappa^{2}+\kappa$ as in the previous lemma and $\eta=\kappa^{2}(4 \kappa+3)$, the following inequality holds:

$$
\mu\left(B_{i}^{k} \cap \Omega_{k+1}\right)<\frac{(4 \theta \eta)^{D_{\mu}}}{a} \mu\left(B_{i}^{k}\right) .
$$

Consequently, for sufficiently large a, we can obtain that

$$
\mu\left(B_{i}^{k}\right) \leq 2 \mu\left(E_{i}^{k}\right) .
$$

Proof. To prove the claim, we apply Lemma 2.1 with $\eta=\kappa^{2}(4 \kappa+3)$. Then, by part i), we have that, for $\theta=4 \kappa^{2}+\kappa$

$$
\Omega_{k+1} \subset \bigcup_{m} \theta B_{m}^{k+1}
$$

and then

$$
\mu\left(B_{i}^{k} \cap \Omega_{k+1}\right) \leq \sum_{m} \mu\left(B_{i}^{k} \cap \theta B_{m}^{k+1}\right) .
$$

Suppose now that $B_{i}^{k} \cap \theta B_{m}^{k+1} \neq \emptyset$. We claim that $r\left(B_{m}^{k+1}\right) \leq r\left(B_{i}^{k}\right)$. Suppose the contrary; namely, $r\left(B_{m}^{k+1}\right)>r\left(B_{i}^{k}\right)$. Then, by property (2.4), we can see that $B_{i}^{k} \subset \kappa^{2}(4 \kappa+3) B_{m}^{k+1}=\eta B_{m}^{k+1}$. For $B=\eta B_{m}^{k+1}$, part iii) from Lemma 2.1 gives us that the average satisfies

$$
\frac{1}{\mu(B)} \int_{B} f d \mu \leq a^{k}
$$

Now, by the properties of the family $\left\{B_{m}^{k+1}\right\}_{m}$ and the doubling condition of $\mu$, we have that, for $a>(2 \eta)^{D_{\mu}}$,

$$
\frac{1}{\mu\left(\eta B_{m}^{k+1}\right)} \int_{\eta B_{m}^{k+1}} f d \mu>\frac{a^{k+1}}{(2 \eta)^{D_{\mu}}}>a^{k} .
$$

This last inequality contradicts (2.16). Then, whenever $B_{i}^{k} \cap \theta B_{m}^{k+1} \neq \emptyset$, we have that $r\left(B_{m}^{k+1}\right) \leq r\left(B_{i}^{k}\right)$ and from that it follows that $B_{m}^{k+1} \subset \eta B_{i}^{k}$. The sum (2.15) now becomes

$$
\begin{aligned}
\mu\left(B_{i}^{k} \cap \Omega_{k+1}\right) & \leq \sum_{m: B_{m}^{k+1} \subset \eta B_{i}^{k}} \mu\left(B_{j}^{k} \cap \theta B_{m}^{k+1}\right) \\
& \leq(2 \theta)^{D_{\mu}} \sum_{m: B_{m}^{k+1} \subset \eta B_{i}^{k}} \mu\left(B_{m}^{k+1}\right) \\
& \leq \frac{(2 \theta)^{D_{\mu}}}{a^{k+1}} \int_{\eta B_{i}^{k}} f d \mu
\end{aligned}
$$


since the sets $\left\{B_{m}^{k+1}\right\}_{m}$ are pairwise disjoint. Finally, by part iii) of Lemma 2.1, we obtain

$$
\mu\left(B_{i}^{k} \cap \Omega_{k+1}\right) \leq \frac{(4 \theta \eta)^{D_{\mu}}}{a} \mu\left(B_{i}^{k}\right),
$$

which is inequality (2.13).

\section{Proofs of the main Results}

Here we present the proof of our main results. Our starting point is a version of the sharp two weight inequality (1.2) valid for SHT from Kail:

Theorem 3.1 ([Kai]). Let $(\mathcal{S}, \rho, \mu)$ be an SHT. Then the $H-L$ maximal operator $M$ defined by (1.9) satisfies the bound

$$
\|M(f \sigma)\|_{L^{p}(w)} \leq C p^{\prime}[w, \sigma]_{S_{p}}\|f\|_{L^{p}(\sigma)},
$$

where $[w, \sigma]_{S_{p}}$ is the Sawyer's condition with respect to balls:

$$
[w, \sigma]_{S_{p}}:=\sup _{B}\left(\frac{1}{\sigma(B)} \int_{B} M\left(\sigma \chi_{B}\right)^{p} w d \mu\right)^{1 / p} .
$$

We now present the proof of the main result.

Proof of Theorem 1.3. By Theorem 3.1, we only need to prove that

$$
[w, \sigma]_{S_{p}} \leq C[w, \sigma, \Phi]_{A_{p}}^{1 / p}[\sigma, \bar{\Phi}]_{W_{p}}^{1 / p}
$$

for some constant $C$, for any Young function $\Phi$, for any $1<p<\infty$. Let $B$ be a fixed ball $B$ and consider the sets $\Omega_{k}$ from (2.12) for the function $\sigma \chi_{B}$ for any $k \in \mathbb{Z}$. Here we remark that in order to apply a $\mathrm{C}-\mathrm{Z}$ decomposition of these sets, we need the level of the decomposition to be larger than the average over the ball. We proceed as follows. Take any $a>1$ and consider $k_{0} \in \mathbb{Z}$ such that

$$
a^{k_{0}-1}<f_{B} \sigma d \mu \leq a^{k_{0}} .
$$

Now, let $A$ be the set of the small values of the maximal function:

$$
A=\left\{x \in B: M\left(\sigma \chi_{B}\right) \leq a f_{B} \sigma d \mu\right\} .
$$

For any $x \in B \backslash A$, we have that

$$
M\left(\sigma \chi_{B}\right)(x)>a f_{B} \sigma d \mu>a^{k_{0}} \geq f_{B} \sigma d \mu .
$$

Therefore,

$$
\begin{aligned}
\int_{B} M\left(\sigma \chi_{B}\right)^{p} w d \mu & =\int_{A} M\left(\sigma \chi_{B}\right)^{p} w d \mu+\int_{B \backslash A} M\left(\sigma \chi_{B}\right)^{p} w d \mu \\
& \leq a^{p} w(B)\left(f_{B} \sigma d \mu\right)^{p}+\sum_{k \geq k_{0}} \int_{\Omega_{k} \backslash \Omega_{k+1}} M\left(\sigma \chi_{B}\right)^{p} w d \mu \\
& =I+I I .
\end{aligned}
$$


The first term $I$ can be bounded easily. By the general Hölder inequality (2.7), we obtain

$$
\begin{aligned}
I & \leq 2 a^{p}\left(f_{B} w d \mu\right)\left\|\sigma^{1 / p^{\prime}}\right\|_{\Phi, B}^{p}\left\|\sigma^{1 / p}\right\|_{\bar{\Phi}, B}^{p} \mu(B) \\
& \leq 2[w, \sigma, \Phi]_{A_{p}} \int_{B} M_{\bar{\Phi}}\left(\sigma^{1 / p} \chi_{B}\right)^{p} d \mu .
\end{aligned}
$$

Now, for the second term $I I$, we first note that

$$
\begin{aligned}
\int_{B \backslash A} M\left(\sigma \chi_{B}\right)^{p} w d \mu & =\sum_{k \geq k_{0}} \int_{\Omega_{k} \backslash \Omega_{k+1}} M\left(\sigma \chi_{B}\right)^{p} w d \mu \\
& \leq a^{p} \sum_{k \geq k_{0}} a^{k p} w\left(\Omega_{k}\right) .
\end{aligned}
$$

By the choice of $k_{0}$, we can apply Lemma 2.1 to perform a $\mathrm{C}-\mathrm{Z}$ decomposition at all levels $k \geq k_{0}$ and obtain a family of balls $\left\{B_{i}^{k}\right\}_{i, k}$ with the properties listed in that lemma. Then,

$$
\begin{aligned}
\int_{B \backslash A} M\left(\sigma \chi_{B}\right)^{p} w d \mu & \leq a^{p} \sum_{k, i}\left(f_{B_{i}^{k}} \sigma \chi_{B} d \mu\right)^{p} w\left(\theta B_{i}^{k}\right) \\
& \leq a^{p} \sum_{k, i}\left(\frac{\mu\left(\theta B_{i}^{k}\right)}{\mu\left(B_{i}^{k}\right)} f_{\theta B_{i}^{k}} \sigma^{\frac{1}{p}} \sigma^{\frac{1}{p^{\prime}}} \chi_{B} d \mu\right)^{p} w\left(\theta B_{i}^{k}\right) .
\end{aligned}
$$

We now proceed as before, using the local generalized Hölder inequality (2.7) and the doubling property (2.3) of the measure (twice). Then we obtain

$$
\int_{B \backslash A} M\left(\sigma \chi_{B}\right)^{p} w d \mu \leq 2 a^{p}(2 \theta)^{(p+1) D_{\mu}}[w, \sigma, \Phi]_{A_{p}} \sum_{k, i}\left\|\sigma^{\frac{1}{p}} \chi_{B}\right\|_{\bar{\Phi}, \theta B_{i}^{k}}^{p} \mu\left(B_{i}^{k}\right) .
$$

The key here is to use Lemma 2.2 to pass from the family $\left\{B_{i}^{k}\right\}$ to the pairwise disjoint family $\left\{E_{i}^{k}\right\}$. Then, for $a \geq 2(4 \theta \eta)^{D_{\mu}}$, we can bound the last sum as follows:

$$
\begin{aligned}
\sum_{k, i}\left\|\sigma^{\frac{1}{p}} \chi_{B}\right\|_{\bar{\Phi}, \theta B_{i}^{k}}^{p} \mu\left(B_{i}^{k}\right) & \leq 2 \sum_{k, i}\left\|\sigma^{\frac{1}{p}} \chi_{B}\right\|_{\bar{\Phi}, \theta B_{i}^{k}}^{p} \mu\left(E_{i}^{k}\right) \\
& \leq 2 \sum_{k, i} \int_{E_{i}^{k}} M_{\bar{\Phi}}\left(\sigma^{\frac{1}{p}} \chi_{B}\right)^{p} d \mu \\
& \leq 2 \int_{B} M_{\bar{\Phi}}\left(\sigma^{\frac{1}{p}} \chi_{B}\right)^{p} d \mu
\end{aligned}
$$

since the sets $\left\{E_{k, j}\right\}$ are pairwise disjoint. Collecting all previous estimates and dividing by $\sigma(B)$, we obtain the desired estimate

$$
[w, \sigma]_{S_{p}}^{p} \leq 4 a^{p}(2 \theta)^{(p+1) D_{\mu}}[w, \sigma, \Phi]_{A_{p}}[\sigma, \bar{\Phi}]_{W_{p}},
$$

and the proof of Theorem 1.3 is complete.

It remains to prove Corollary 1.4. To that end, we need to consider the special case of $\Phi(t)=t^{p^{\prime}}$. 
Proof of Corollary 1.4. Considering then $\Phi(t)=t^{p^{\prime}}$, the quantity (1.7) is

$$
\begin{aligned}
A_{p}(w, \sigma, B, \Phi) & =\left(f_{B} w d \mu\right)\left\|\sigma^{1 / p^{\prime}}\right\|_{\Phi, B}^{p} \\
& =\left(f_{B} w(y) d \mu\right)\left(f_{B} \sigma d \mu\right)^{p-1} .
\end{aligned}
$$

In addition, we have from (1.8) that $\left[\sigma, \overline{\Phi_{p^{\prime}}}\right]_{W_{p}}=\left[\sigma, \Phi_{p}\right]_{W_{p}}=[\sigma]_{A_{\infty}}$ and therefore we obtain (1.11).

For the proof of Corollary 1.5, we simply use the boundedness of $M_{\bar{\Phi}}$ on $L^{p}(\mu)$,

$$
[\sigma, \bar{\Phi}]_{W_{p}}:=\sup _{B} \frac{1}{\sigma(B)} \int_{B} M_{\bar{\Phi}}\left(\sigma^{1 / p} \chi_{B}\right)^{p} d \mu \leq\left\|M_{\bar{\Phi}}\right\|_{L^{p}}^{p} .
$$

The proof of Corollary 1.6 is trivial.

\section{Appendix}

Here we include a direct proof of a version of Corollary 1.5 which is better in terms of the dependence on $p$. Precisely, we have the following proposition.

Proposition 4.1. Let $1<p<\infty$. For any pair of weights $w, \sigma$ and any Young function $\Phi$, there exists a structural constant $C>0$ such that

$$
\|M(f \sigma)\|_{L^{p}(w)} \leq C[w, \sigma, \Phi]_{A_{p}}^{1 / p}\left\|M_{\bar{\Phi}}\right\|_{L^{p}}\|f\|_{L^{p}(\sigma)} .
$$

Proof of Proposition 4.1. By density it is enough to prove the inequality for each non-negative bounded function with compact support $f$. We first consider the case of unbounded $S$. In this case we have $f_{S} f \sigma d \mu=0$. Therefore, instead of the sets from (2.12), we consider

$$
\Omega_{k}=\left\{x \in \mathcal{S}: M(f \sigma)(x)>a^{k}\right\},
$$

for any $a>1$ and any $k \in \mathbb{Z}$. Then, we can write

$$
\int_{\mathcal{S}} M(f \sigma)^{p} w d \mu=\sum_{k} \int_{\Omega_{k} \backslash \Omega_{k+1}} M(f \sigma)^{p} w d \mu .
$$

Then, following the same line of ideas as in the proof of Theorem 1.3, we obtain

$$
\int_{\mathcal{S}} M(f \sigma)^{p} w d \mu \leq 2 a^{p}(2 \theta)^{(p+1) D_{\mu}}[w, \sigma, \Phi]_{A_{p}} \sum_{k, i}\left\|f \sigma^{\frac{1}{p}}\right\|_{\bar{\Phi}, \theta B_{i}^{k}}^{p} \mu\left(B_{i}^{k}\right) .
$$

By Lemma 2.2 we can replace the family $\left\{B_{i}^{k}\right\}$ by the pairwise disjoint family $\left\{E_{i}^{k}\right\}$ to obtain the desired estimate:

$$
\int_{\mathcal{S}} M(f \sigma)^{p} w d \mu \leq 4 a^{p}(2 \theta)^{(p+1) D_{\mu}}[w, \sigma, \Phi]_{A_{p}}\left\|M_{\bar{\Phi}}\right\|_{L^{p}}^{p} \int_{S} f^{p} \sigma d \mu .
$$

In the bounded case, the whole space is a ball and we can write $\mathcal{S}=B(x, R)$ for any $x$ and some $R>0$. The problem here is to deal with the small values of $\lambda$, since we cannot apply Lemma 2.2 for $a^{k} \leq f_{S} f \sigma d \mu$. We then take any $a>1$ and consider $k_{0} \in \mathbb{Z}$ to verify (3.3):

$$
a^{k_{0}-1}<f_{S} f \sigma d \mu \leq a^{k_{0}}
$$

and argue as in the proof of Theorem 1.3 . 
Now, from this last proposition, we can derive another proof of the mixed bound (1.11) from Corollary 1.4. The disadvantage of this approach with respect to the previous one is that we need a deep property of $A_{\infty}$ weights: the sharp Reverse Hölder Inequality. In the whole generality of SHT, we only know a weak version of this result from the recent paper [HPR12]:

Theorem 4.2 (Sharp weak Reverse Hölder Inequality, [HPR12]). Let $w \in A_{\infty}$. Define the exponent $r(w)=1+\frac{1}{\tau_{\kappa \mu}[w]_{A_{\infty}}}$, where $\tau_{\kappa \mu}$ is a structural constant. Then,

$$
\left(f_{B} w^{r(w)} d \mu\right)^{1 / r(w)} \leq 2(4 \kappa)^{D_{\mu}} f_{2 \kappa B} w d \mu,
$$

where $B$ is any ball in $\mathcal{S}$.

The other ingredient for the alternative proof of Corollary 1.4 is the known estimate for the operator norm for $M$. For any $1<q<\infty$, we have that $\|M\|_{L^{q}}^{q} \sim$ $q^{\prime}$.

Another proof of Corollary 1.4. Consider the particular choice of $\Phi(t)=t^{p^{\prime} r}$ for $r>1$. Then quantity (1.7) is

$$
A_{p}(w, \sigma, B, \Phi)=\left(f_{B} w(y) d \mu\right)\left(f_{B} \sigma^{r} d \mu\right)^{p / r p^{\prime}} .
$$

If we choose $r$ from the sharp weak reverse Hölder property (Theorem 4.2), we obtain that

$$
\begin{aligned}
A_{p}(w, \sigma, B, \Phi) & =\left(f_{B} w d \mu\right)\left(2(4 \kappa)^{D_{\mu}} f_{2 \kappa B} \sigma d \mu\right)^{p-1} \\
& \leq 2^{p-1}(4 \kappa)^{p D_{\mu}}\left(f_{2 \kappa B} w d \mu\right)\left(f_{2 \kappa B} \sigma d \mu\right)^{p-1} \\
& \leq 2^{p-1}(4 \kappa)^{p D_{\mu}}[w, \sigma]_{A_{p}} .
\end{aligned}
$$

Therefore the proof of Proposition 4.1 gives

$$
\|M(f \sigma)\|_{L^{p}(w)} \leq C[w, \sigma]_{A_{p}}^{1 / p}\left\|M_{\bar{\Phi}}\right\|_{L^{p}(\mathcal{S}, d \mu)}\|f\|_{L^{p}(\sigma)} .
$$

We conclude with the proof by computing $\left\|M_{\bar{\Phi}}\right\|_{L^{p}}$ for $\Phi(t)=t^{p^{\prime} r}$. We use (2.9), and then we obtain that $\left\|M_{\bar{\Phi}}\right\|_{L^{p}}^{p} \leq c r^{\prime} p^{\prime}$. But, by the choice of $r$, it follows that $r^{\prime} \sim[\sigma]_{A_{\infty}}$ and we obtain (1.11).

\section{REFERENCES}

[Aim85] Hugo Aimar, Singular integrals and approximate identities on spaces of homogeneous type, Trans. Amer. Math. Soc. 292 (1985), no. 1, 135-153, DOI 10.2307/2000174. MR:805957 (86m:42022)

[AM84] Hugo Aimar and Roberto A. Macías, Weighted norm inequalities for the HardyLittlewood maximal operator on spaces of homogeneous type, Proc. Amer. Math. Soc. 91 (1984), no. 2, 213-216, DOI 10.2307/2044629. MR740173 (85m:42019)

[BR] Oleksandra Beznosova and Alexander Reznikov, Equivalent definitions of dyadic Muckenhoupt and reverse Hölder classes in terms of Carleson sequences, weak classes, and comparability of dyadic $L \log L$ and $A_{\infty}$ constants. Preprint, arXiv:1201.0520 (2012). 
[DMRO13] Javier Duoandikoetxea, Francisco Martín-Reyes, and Sheldy Ombrosi, On the $A_{\infty}$ conditions for general bases. 2013. Private communication.

[Fuj78] Nobuhiko Fujii, Weighted bounded mean oscillation and singular integrals, Math. Japon. 22 (1977/78), no. 5, 529-534. MR0481968 (58 \#2058)

[GCRdF85] José García-Cuerva and José L. Rubio de Francia, Weighted norm inequalities and related topics, North-Holland Mathematics Studies, vol. 116, North-Holland Publishing Co., Amsterdam, 1985. Notas de Matemática [Mathematical Notes], 104. MR807149 (87d:42023)

[GGKK98] Ioseb Genebashvili, Amiran Gogatishvili, Vakhtang Kokilashvili, and Miroslav Krbec, Weight theory for integral transforms on spaces of homogeneous type, Pitman Monographs and Surveys in Pure and Applied Mathematics, vol. 92, Longman, Harlow, 1998. MR 1791462 (2003b:42002)

[HP] Tuomas Hytönen and Carlos Pérez, Sharp weighted bounds involving $A_{\infty}$, Anal. PDE 6 (2013), no. 4, 777-818, DOI 10.2140/apde.2013.6.777. MR3092729

[HPR12] Tuomas Hytönen, Carlos Pérez, and Ezequiel Rela, Sharp reverse Hölder property for $A_{\infty}$ weights on spaces of homogeneous type, J. Funct. Anal. 263 (2012), no. 12, 3883-3899, DOI 10.1016/j.jfa.2012.09.013. MR2990061

[Hru84] Sergei V. Hruščev, A description of weights satisfying the $A_{\infty}$ condition of Muckenhoupt, Proc. Amer. Math. Soc. 90 (1984), no. 2, 253-257, DOI 10.2307/2045350. MR.727244 (85k:42049)

[Kai] Anna Kairema, Two-weight norm inequalities for potential type and maximal operators in a metric space, Publ. Mat. 57 (2013), no. 1, 3-56, DOI 10.5565/PUBLMAT_57113_01. MR3058926

[LL] Liguang Liu and Teresa Luque. A $B_{p}$ condition for the strong maximal function. Trans. Amer. Math. Soc. (to appear).

[LM] Andrei K. Lerner and Kabe Moen. Mixed $A_{p}-A_{\infty}$ estimates with one supremum. Preprint, arXiv:1212.0571 (2013).

[Moe09] Kabe Moen, Sharp one-weight and two-weight bounds for maximal operators, Studia Math. 194 (2009), no. 2, 163-180, DOI 10.4064/sm194-2-4. MR2534183 (2011h:42024)

[MP] Mieczysław Mastyło and Carlos Pérez, The maximal operators between banach function spaces, Indiana Univ. Math. J. (To appear).

[MS79] Roberto A. Macías and Carlos Segovia, Lipschitz functions on spaces of homogeneous type, Adv. in Math. 33 (1979), no. 3, 257-270, DOI 10.1016/0001-8708(79)90012-4. MR.546295 (81c:32017a)

[Neu83] C. J. Neugebauer, Inserting $A_{p}$-weights, Proc. Amer. Math. Soc. 87 (1983), no. 4, 644-648, DOI 10.2307/2043351. MR687633 (84d:42026)

[Pér95] C. Pérez, On sufficient conditions for the boundedness of the Hardy-Littlewood maximal operator between weighted $L^{p}$-spaces with different weights, Proc. London Math. Soc. (3) 71 (1995), no. 1, 135-157, DOI 10.1112/plms/s3-71.1.135. MR.1327936 (96k:42023)

[PS04] Gladis Pradolini and Oscar Salinas, Maximal operators on spaces of homogeneous type, Proc. Amer. Math. Soc. 132 (2004), no. 2, 435-441 (electronic), DOI 10.1090/S0002-9939-03-07079-5. MR2022366 (2004j:42013)

[PW01] Carlos Pérez and Richard L. Wheeden, Uncertainty principle estimates for vector fields, J. Funct. Anal. 181 (2001), no. 1, 146-188, DOI 10.1006/jfan.2000.3711. MR.1818113(2002h:42035)

[Saw82] Eric T. Sawyer, A characterization of a two-weight norm inequality for maximal operators, Studia Math. 75 (1982), no. 1, 1-11. MR676801 (84i:42032)

[SW92] E. Sawyer and R. L. Wheeden, Weighted inequalities for fractional integrals on Euclidean and homogeneous spaces, Amer. J. Math. 114 (1992), no. 4, 813-874, DOI 10.2307/2374799. MR1175693 (94i:42024) 
[Wil87] J. Michael Wilson, Weighted inequalities for the dyadic square function without dyadic $A_{\infty}$, Duke Math. J. 55 (1987), no. 1, 19-50, DOI 10.1215/S0012-7094-8705502-5. MR883661 (88d:42034)

Departamento de Análisis Matemático, Facultad de Matemáticas, Universidad de Sevilla, 41080 Sevilla, Spain

E-mail address: carlosperez@us.es

Departamento de Análisis Matemático, Facultad de Matemáticas, Universidad de Sevilla, 41080 Sevilla, Spain

E-mail address: erela@us.es 